\title{
9 AVAliAção dA SAÚdE MENTAL POSItIVA dE DISCENTES DE ENFERMAGEM
}

\author{
$\mid$ Michelle Ferreira $^{1}$; Elaine Cortez ${ }^{2}$; Jorge da Silva ${ }^{3}$; Maylu Júlio Ferreira ${ }^{4} \mid$
}

\section{RESUMO}

CONTEXTO: A importância da saúde mental é reconhecida pela Organização Mundial de Saúde (OMS), na sua própria definição de saúde, como um estado de completo bem-estar físico, mental e social.

OBJETIVO(S): O objetivo geral foi avaliar a saúde mental positiva dos discentes de enfermagem por meio do Questionário de Saúde Mental Positiva (QSM+) de Lluch (2003) traduzido e adaptado para o português (Carvalho \& Sequeira, 2009) que visa avaliar a capacidade do aluno em termos de gestão das variáveis positivas que integram a saúde mental.

METODOLOGIA: Trata-se de pesquisa quantitativa exploratória, que teve como sujeitos 269 acadêmicos de enfermagem. Foi traçado inicialmente o perfil dos sujeitos da pesquisa com finalidade de agrupar e conhecer a população.

RESULTADOS: Com base nos objetivos foi evidenciado que os acadêmicos de enfermagem, em sua maioria, estão satisfeitos com sua vida pessoal, são bons ouvintes, tem dificuldade para lidar com o stress, pouco confiantes em si mesmos, menos da metade se diz disposta a ajudar o próximo, possuem facilidade para estabelecer relações interpessoais.

CONCLUSÕES: Há necessidade de se investir em estratégias que viabilizem promover a saúde do acadêmico de enfermagem, durante sua formação, para que na vida profissional tenham melhor equilíbrio diante das situações impostas pelo ambiente de trabalho, bem como a necessidade de conscientização da importância do cuidado.

\section{PALAVRAS-CHAVE: Discentes; Saúde mental; Enfermagem}

\section{RESUMEN}

\section{"Evaluación de lasalud mental positiva de losestudiantes de en- fermaria"}

CONTEXTO: La importancia de la salud mental es reconocida por la Organización Mundial de la Salud(OMS), en su propia definición de la salud como un estado de bienestar físico, mental y social.

OBJETIVO(S): El objetivo general fue evaluar la salud mental positiva de los estudiantes de enfermería a través del Cuestionario de Salud Mental Positiva (QSM+) de Lluch (2003) ha sido traducido y adaptado para el portugués (Carvalho y Sequeira, 2009) diseñado para evaluar la capacidad del alumno en cuanto a la gestión de las variables positivas que integran la salud mental.

METODOLOGÍA: Se trata de una investigación exploratoria y cuantitativa que tuvo como el tema 269 estudiantes de enfermería. Se trazó inicialmente el perfil de los sujetos de investigación con el fin de agrupar y conocer a la gente.

RESULTADOS: Sobre la base de los objetivos se evidenció que los estudiantes de enfermería, en su mayoría, están satisfechos con su vida personal, son buenos oyentes, tienen dificultades para hacer frente al estrés, la falta de confianza en sí mismos, menos de la mitad se dice dispuesto a ayudar a continuación, tienen facilidad para establecer relaciones interpersonales.

CONCLUSIONES: Hay necesidad de invertir en estrategias que permitan promover la salud de los estudiantes de enfermería durante su formación, por lo que la vida profesional tiene un mejor equilibrio en la cara de situaciones impuestas por el ambiente de trabajo y la necesidad de tomar conciencia de la importancia de la atención.

\section{DESCRIPTORES: Estudiantes; Salud mental; Enfermería}

\begin{abstract}
“The positive mental health evaluation of nursing students"

BACKGROUND: The importance of mental health is recognized by the World Health Organization (WHO) in its own definition of health as a state of complete physical, mental and social well-being.

AIM:The overall objective was to evaluate the positive mental health of nursing students through the Positive Mental Health Questionnaire (QSM +) of Lluch (2003), already translated and adapted to Portuguese (Carvalho\&Sequeira, 2009) that aims to assess the student's ability in terms of management of positive variables that integrate mental health.

METHODS: This is a quantitative exploratory research, which took as subjects 269 nursing students. It was initially traced the profile of the research subjects with a purpose of grouping and understand the population involved in the research.

RESULTS: Based on the quantitative targets we showed that nursing students mostly are satisfied with their personal life, are good listeners, have trouble dealing with stress, lack of self confidence, unwilling to help others, and have facility to establish interpersonal relationships.

CONCLUSIONS: Thus, we conclude that it's necessary to invest in strategies to promote nursing student health during training allowing them to have better balance against situations imposed by the work, as well as the consciousness regarding the importance of care.
\end{abstract}

\section{KEYWORDS: Leaners; Mental health; Quality health}

Submetido em 22-02-2016

Aceite em 30-05-2016

1 Fisioterapeuta; Enfermeira pela Universidade Federal Fluminense, Escola de Enfermagem Aurora de Afonso Costa, Niterói - RJ, Brasil, mi.ju@hotmail.com

2 Enfermeira; Pós-Doutora em Enfermagem/Saúde Mental; Professora naUniversidade Federal Fluminense,Departamento Materno-Infantil e Psiquiatria, 24020-091 Niterói RJ, Brasil, nanicortez@hotmail.com

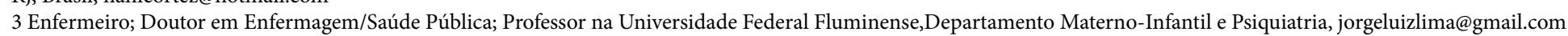
4 Bolsista do Programa Institucional de Bolsas de Iniciação Científica; Acadêmica de Enfermagem na Universidade Federal Fluminense, Brasil, maylus2@gmail.com 


\section{INTRODUÇÃO}

A saúde mental está na agenda da saúde pública de todo o mundo. Sabe-se hoje que as perturbações mentais afetam todas as faixas etárias e são responsáveis por elevados custos sociais e econômicos (Comissão Nacional para a Reestruturação dos Serviços de Saúde Mental, 2007). Dessa forma, foi realizado uma revisão sobre o tema onde se evidenciou a necessidade deste estudo pela constatação de poucos estudos realizados.

Na busca realizada na base de dados da Biblioteca Virtual em Saúde (BVS), utilizaram-se os Descritores em Ciências da Saúde (DeCS): "discentes, saúde mental e enfermagem", aceitando-se apenas textos disponíveis na íntegra, nos idiomas português e inglês e estudos com menos de 10 anos de publicação, excluindo-se todos aqueles que não incluíam nesse perfil de busca, foram encontrados 39 trabalhos publicados, entre teses e artigos. Entre esses, apenas 5 tratavam sobre a saúde mental dos estudantes universitários, com destaque para o artigo intitulado Avaliação da saúde mental positiva em estudantes do ensino superior (Sequeira et al, 2009), o qual objetivou avaliar os níveis de saúde mental em estudantes do ensino superior e correlacionar a saúde mental com a morbilidade psiquiátrica referida pelos estudantes, através dos Questionário de Saúde mental positiva - QSM+ (Lluch, 2003) e Inventário de Saúde Mental - ISM (Ribeiro, 2001). Como conclusão verificou-se elevada correlação negativa entre ambos os instrumentos utilizados o que reforça a necessidade de intervenção em nível de promoção da saúde mental como estratégia de prevenção a morbilidade psiquiátrica.

Também, dentre os estudos encontrados, destacam-se o de Castillo\& Schwartz (2013) e o de Eisenberg, Gollust, Golberstein, \& Hefner (2007), que afirmam que no Ensino Superior (ES) o número de jovens estudantes com perturbações mentais tem aumentado progressivamente, assim como a própria severidade dos problemas psicológicos evidenciados pelos estudantes que procuram ajuda nos serviços de aconselhamento disponibilizados nas Instituições de Ensino Superior (IES) (Hunt \&Eisenberg, 2010).

Nesse sentido, o contexto do ensino superior proporciona ao estudante uma série de desafios, tanto pessoais quanto profissionais a qual exige capacidades e habilidades talvez não utilizadas anteriormente.

Sabe-se do impacto de determinadas situações de vida na condição de saúde das pessoas.
Estudos têm demonstrado a influência, por exemplo, do contexto universitário, e as mudanças que o mesmo deflagra na vida do indivíduo e na saúde mental dos jovens (Giglio, 1976; Adewuya, 2006;). Além disso, o impacto de determinadas situações de vida - que podem ou não configurar uma crise - têm um reflexo importante na saúde, física ou psicológica dos indivíduos (Dias, Rubin, Dias e Gauer, 2007).

De acordo com os dados obtidos pelo National Survey of Counseling Center Directors (Gallagher, 2013), está patente um aumento substancial de casos de alunos com problemas psicológicos severos (82\%), situações de crise que requerem uma resposta imediata (80\%), questões relacionadas com a medicação (73\%), dificuldades de aprendizagem (61\%), uso de drogas ilícitas (52\%), automutilações (48\%), agressões sexuais no campus (40\%), abuso de álcool (34\%), perturbações alimentares (34\%) e preocupações com o futuro profissional (30\%).

Diante desta revisão, observa-se a necessidade de que mais estudos envolvendo esta população sejam desenvolvidos na realidade brasileira, e que os mesmos produzam mais conhecimentos sobre novas formas de intervenção para a promoção da saúde, no contexto universitário. Deste modo, esta pesquisa torna-se relevante, pois estudo sobre saúde mental de estudantes universitários requer investigação que considere o contexto do ensino superior, e as questões pertinentes para que mais intervenções de promoção da saúde mental sejam elaboradas e executadas de acordo com a realidade.

Ressalta-se que, saúde mental é definida como o estado de bem-estar no qual o indivíduo percebe as próprias habilidades, pode lidar com os estresses normais da vida, é capaz de trabalhar produtivamente e está apto a contribuir com sua comunidade. É mais do que ausência de doença mental, e não existe saúde sem saúde mental (Organização Mundial de Saúde [OMS], 2002). Numa perspectiva holística, a saúde mental inclui a capacidade do indivíduo para apreciar a vida e procurar o equilíbrio entre as atividades, e os esforços para atingir a resiliência psicológica (Sá, 2010).

Diante desse contexto, o estudo teve como objetivos gerais avaliar a saúde mental positiva dos discentes de enfermagem em termos de satisfação pessoal, atitude positiva, autocontrole, autonomia, capacidade de resolução de problemas e habilidades de relação interpessoal, de acordo com o Questionário de Saúde Mental positiva (QSM+) de Lluch (2003), traduzido e adaptado para o português (Carvalho e Sequeira, 2009). 


\section{METODOLOGIA}

Trata-se de estudo com abordagem quantitativa, pois se pretendeu recolher e tratar de forma sistematizada e estatística, estabelecendo relações entre variáveis no intuito de responder às questões de investigação. Caracteriza-se por ser do tipo transversal, onde os dados recolhidos num só momento, sem existir intervenção. Os dados visaram avaliar a capacidade do aluno em termos de gestão das variáveis positivas que integram a saúde mental.

Ressalta-se que ao escolher a pesquisa de campo, não podemos deixar de abordar sobre os aspectos que envolvem a eticidade da pesquisa, nos termos do que dispõe a Resolução $n^{\circ} 466 / 12$ do Conselho Nacional de Saúde ([CNS], 2006). A pesquisa foi encaminhada à Plataforma Brasil e aprovada pelo Comitê de Ética em Pesquisa da Faculdade de Medicina/Hospital Universitário Antônio Pedro sob o protocolo número: CEP CMM/HUAPE $n^{\circ} 836.513$ em 17/10/2014. Os discentes foram convidados a participar da pesquisa, receberam os esclarecimentos necessários sobre o objetivo da pesquisa, da garantia de sigilo e anonimato, do caráter voluntário da participação, sem incidência de prejuízos. Após tal ciência, os participantes assinaram o Termo de Consentimento Livre e Esclarecido, confirmando sua participação voluntária na pesquisa.

O cenário da pesquisa foi a Escola de Enfermagem Aurora de Afonso Costa (EEAAC/UFF), localizada no município de Niterói (RJ-Brasil). O Curso de Enfermagem da UFF possibilita ao acadêmico uma estreita relação com as questões da saúde, em geral, e da profissão em particular, através de disciplinas ministradas desde o $1^{\circ}$ período, tornando o curso mais atraente para o aluno. A formação do enfermeiro tem caráter generalista, o que possibilita conhecimento amplo da enfermagem no âmbito da assistência, ensino e pesquisa. Tem como objetivo formar um profissional crítico e reflexivo com competência técnico-científica, ético-política, social e educativa, comprometido com a prática social, capaz de identificar as necessidades individuais e coletivas da população, seus determinantes e atuar no processo saúde-doença, garantindo a qualidade da assistência de enfermagem em todos os níveis de atenção à saúde.

Ressalta-se que o curso de Graduação de Enfermagem e Licenciatura da UFF tem carga horária de 5.440 horas, sendo que deste total, $24 \%$ são destinados aos estágios curriculares obrigatórios do campo de formação específica e da licenciatura, respectivamente 900 e 400 horas.
O currículo do curso de enfermagem e suas atividades agregadas exigem dedicação intensa do estudante, havendo uma grade horária com pouco tempo livre. Tal realidade, somada ao excesso de conteúdo ministrado, leva o estudante a diminuir suas horas de sono e apresentar sonolência diurna, que afeta a sua qualidade de vida. Os mesmos passam 4 anos e meio entre vários campos de estudos (Campus Gragoatá, Instituto Biomédico, Valonguinho, EEAAC/UFF, Hospital Universitário e diversos campi) ao longo do dia, visto que o curso se dá em forma de período integral.

Foram considerados sujeitos da pesquisa os discentes do curso de graduação em enfermagem. Ressalta-se que os critérios de inclusão dos sujeitos do estudo foram: discentes matriculados no $2^{\circ}$ semestre do ano de 2014 , ou seja, do $1^{\circ}$ ao $9^{\circ}$ período, excluindo os que se encontravam com a matrícula trancada. De acordo com dados colhidos do site da EEAAC/UFF, constam 580 estudantes matriculados no curso de enfermagem, dessa amostra 269 participaram $(\mathrm{N}=269)$ e $268(\mathrm{~N}=268)$ responderam ao questionário, cursando do $1^{\circ}$ ao $9^{\circ}$ período, de entre os quais com maior participação os discentes do $1^{\circ}$ período $17,1 \%$, seguido pelo $6^{\circ}$ período $16,7 \%$. A técnica utilizada na coleta de dados foi o questionário, colhendo impressões individuais sobre aspectos relacionados aos objetivos do estudo dentre os quais o Questionário de Saúde Mental Positiva (QSM+), desenvolvido inicialmente por Lluch (2003), seguindo os critérios de Saúde Mental Positiva formulados por Jahoda (1958). Foi reformulado e validado por Carvalho e Sequeira(2009), versão portuguesa. Esse questionário propõe um Modelo Multifatorial de Saúde Mental Positiva configurado por seis fatores/dimensões, algo diferente formulado por Jahoda.

O QSM+ trata-se de um questionário com 39 questões que contém uma série de afirmações sobre a forma de pensar, sentir e agir de cada um, agrupadas em seis fatores/dimensões (satisfação pessoal, atitude positiva, autocontrole, autonomia, capacidade de realização de problemas e habilidades de realização interpessoal), que oferece aos inquiridos quatro possibilidades de resposta: sempre ou quase sempre, com bastante frequência, algumas vezes, quase nunca ou nunca.

Os dados recolhidos foram editados pelo investigador em base de dados criada para o análise no programa SPSS (Statistical Package for the Social Sciences) versão 20.0 do Windows. O conjunto das informações recolhidas foi inicialmente analisado, de acordo com a metodologia descritiva usual, após a sua informatização. Apenas foram validados os questionários preenchidos de forma correta e completa. 


\section{RESULTADOS}

O ingresso do estudante no ensino superior traz consigo uma gama de mudanças em nível pessoal, cognitivo, profissional, afetivo e social, além de acarretar uma série de expectativas em relação ao curso de graduação escolhido. Essas expectativas, muitas vezes, estão acompanhadas por ansiedade, medo e dúvidas acerca de como será seu desempenho acadêmico e sua formação profissional. Desse modo, o curso de graduação será o novo ambiente de formação dos estudantes, capaz de interferir de forma positiva ou negativa em sua construção como acadêmico e futuro profissional.

$\mathrm{Na}$ pesquisa feita, realizou-se análise descritiva dos dados onde foram diagnosticados indicadores significativos nos itens em cada dimensão pesquisada, dentre as opções de resposta: sempre ou quase sempre; na maioria das vezes; algumas vezes; raramente ou nunca. $\mathrm{Na}$ dimensão avaliada "satisfação pessoal", a maioria das escolhas feitas pelos estudantes de enfermagem reflete que estão satisfeitos na maioria das vezes com suas escolhas, com seu aspecto físico $(56,1 \%)$, e são otimistas quanto ao seu futuro (71\%), não permitindo que o sentimento negativo seja preponderante em suas vidas. Dela Coleta e Dela Coleta (2006), ao estudarem felicidade e bem-estar subjetivo em uma amostra de 252 estudantes universitários, entre muitos achados, encontraram resultados superiores à média, indicando sentimentos positivos de felicidade, satisfação geral e com diferentes aspectos da vida, gratidão, plenitude, deleite (flow), auto avaliação do rendimento escolar e expectativas futuras, tanto do ponto de vista pessoal quanto profissional. Mostraram também que os sujeitos com níveis mais altos de felicidade, satisfação com a vida, bem-estar subjetivo apresentam mais elevado auto avaliações como estudantes e condutas escolares mais apropriadas, tendo em vista o aproveitamento acadêmico. Pensando na segunda dimensão avaliada, "Atitude Pró-social", observa-se com os resultados encontrados no estudo com os discentes, que se pode afirmar que se mostram pessoas altruístas, bons ouvintes $(76,6 \%)$, preocupam-se em ajudar o próximo (47,2\%). Tal resultado é muito relevante quando se associa esses discentes ao seu campo de estudos. A enfermagem profissional no mundo foi erigida a partir das bases científicas propostas por Florence Nightingale, que foi influenciada diretamente pela sua passagem nos locais onde se executava o cuidado de enfermagem leigo e fundamentado nos conceitos religiosos de caridade, amor ao próximo, doação, humildade, e também pelos preceitos de valorização do ambiente adequado para o cuidado, divisão social do trabalho em enfermagem e autoridade sobre o cuidado a ser prestado.
De acordo com os resultados encontrados na dimensão de "Autocontrole", os discentes de enfermagem demonstram pouco controle emocional diante das adversidades da vida, permitindo que percam seu equilíbrio interior $(52,4 \%)$, demonstrando ansiedade e pouca habilidade para lidar com o stress (46,8\%). Justifica-se o fato de estudarem em período integral, com pouco tempo de lazer, pressões dos pais e da própria instituição de ensino.

Pôde-se perceber, de acordo com os resultados na dimensão "Autonomia", que os discentes são em sua maioria são independentes, capazes de tomar suas próprias decisões apesar da pouca idade, e apesar de estarem distante dos pais no seu cotidiano, mostram-se seguros em suas escolhas (53,9\%), não estão muito preocupados com a opinião que os outros podem ter $(59,1 \%)$ e essa mesma opinião não influencia em suas decisões (44,6\%) e sabe dizer "não" sem problemas (64,7\%).

Pensando na dimensão "Resolução de problemas e Realização pessoal" e analisando os resultados encontrados em nossa pesquisa, pode-se dizer que os discentes de enfermagem encontram problemas e limitações para gerenciar seus próprios problemas, onde não possuem habilidade para adaptarem-se as mudanças em sua vida. Não se preocupam em sua evolução pessoal, não se importado em melhorar como pessoa. Contudo, o mais preocupante é o fato dos dados terem demonstrado que a maioria $(72,1 \%)$ não gosta de ajudar o próximo. Fato preocupante, uma vez que a universidade visa formar futuros enfermeiros, cuidadores de seres humanos que necessitam de atenção e carinho.

Por fim, na dimensão "Habilidades de relação interpessoal”, em sua grande maioria como demonstrado anteriormente, os discentes tem facilidade para estabelecer relações interpessoais $(56,1 \%)$, para dar apoio emocional $(62,5 \%)$ e conseguem entender o conceito de hierarquia, pois tendem a ter uma boa relação com seus superiores. $\mathrm{O}$ que muito nos preocupa é que esses mesmos jovens não se consideram pessoas sociáveis $(46,1 \%)$ e não se acham bons conselheiros (42,8\%). Dada questão deve-se ao fato ainda serem imaturos e pouco experientes, o que os impede de pensar que podem ajudar seu próximo com seus conhecimentos e socializar para trocar experiências. Mas em um contexto geral, pode-se observar que são jovens dispostos a estabelecer relações interpessoais, manter relações com maior intimidade, que buscam melhorar em sua vida, pois sabem que o networking é de extrema importância no mundo competitivo em que vivemos. Em suma, observou-se nos resultados que a maioria dos discentes de enfermagem é do sexo feminino, com idade entre 18 e 47 anos. 
Nos dados obtidos em cada dimensão pesquisada, em termos positivos, encontram-se satisfeitos com sua vida pessoal, veem seu futuro com otimismo, se consideram pessoas importantes e úteis, são bons ouvintes, entendem bem os sentimentos dos outros, preocupam-se pouco com críticas recebidas, tem facilidade para estabelecer relações interpessoais, principalmente quando se trata de pessoas superiores em seus cargos (chefia/ professores). Em contrapartida, evidenciaram-se dados importantes que destacam um aspecto negativo e preocupante. Em sua maioria encontramos discentes que não sabem lidar com o estresse, são ainda muito dependentes para tomar suas próprias decisões, inseguros, não procuram melhorar como pessoa, não são dignos de confiança, apresentam dificuldades para arranjar soluções para seus problemas, e não gostam de ajudar o próximo quando o mesmo necessita.

\section{DISCUSSÃO}

A entrada no ensino superior constitui, para muitos jovens, uma situação de transição acompanhada por um conjunto de mudanças pessoais e sociais, com implicações ao nível da sua saúde mental. A saúde mental do estudante de enfermagem deverá ser uma prioridade e constituir um foco de atenção das instituições de ensino superior em articulação com os serviços de saúde.

O questionário de Saúde Mental Positiva revelou-se com um instrumento importante para avaliar a saúde mental dos estudantes do ensino superior. Trata-se também de um instrumento de grande relevância para a prática clínica, na medida em que permite avaliar o potencial das pessoas para lidar com as adversidades e, por outro lado, permite identificar as pessoas com maior vulnerabilidade mental. O presente estudo oferece uma nova visão acerca da importância da avaliação da Saúde Mental Positiva, integrada numa lógica de Promoção da Saúde Mental, defendida como prioritária, há vários anos, por diversas organizações de renome internacional. Esses dados alertam para a necessidade de intervenção ao nível da promoção da saúde mental dos estudantes de enfermagem, essencialmente ao nível da saúde mental positiva, intervindo na melhoria da autoestima, na promoção da autonomia, no desenvolvimento de habilidades de resolução de problemas, de forma a ser efetivo na prevenção da ansiedade e da depressão.

Precisamos investir em estratégias que viabilizem promover a saúde do acadêmico de enfermagem durante sua formação para que na vida profissional tenham um maior equilíbrio diante das situações impostas pelo ambiente onde irão atuar e a necessidade de conscientização da importância do cuidado.

\section{CONCLUSÃO}

Conclui-se que precisamos investir em estratégias que viabilizem promover a saúde do acadêmico de Enfermagem durante sua formação para que, na sua vida profissional, tenham um maior equilíbrio diante das situações impostas pelo ambiente de trabalho e sejam conscientes acerca da importância do cuidado. Também como proposta, pensou-se na implementação de projetos de apoio emocional e cognitivo, objetivando promover suporte profissional suficiente para prevenir, reduzir ou mesmo cessar situações de stress. Desta forma, busca-se a melhora da qualidade de vida dos discentes, uma vez que são os futuros profissionais que estarão à frente do atendimento a clientes mais ou tão vulneráveis quanto eles. Torna-se viável ainda, pensar em uma reformulação das disciplinas que são feitas fora do Campus da Enfermagem. É desgastante para os estudantes a locomoção de um campus ao outro, às vezes com calor em demasia, chuva, refeições rápidas para chegar a tempo nas aulas e a distância percorrida que chega a ser de quilômetros entre um campus e outro. As disciplinas deveriam concentrar-se em um mesmo local, com professores voltados ao ensino na Escola de Enfermagem, o que facilitaria a dinâmica do dia, já que o curso é tido em tempo integral.

É importante que este caminho seja dado como opção no nosso ambiente de estudo para que possibilite um crescimento não somente como futuros profissionais, mas como pessoas. Compreender e trabalhar o indivíduo e/ou coletivo em busca de um ser que desenvolve estratégias de enfrentamento de sucesso capazes de garantir-lhe a saúde integral e conquista de seus objetivos pessoais, de cidadão e profissional.

Assim, é importante que a universidade esteja consciente de seu papel na formação não somente técnica, mas também sociocultural do enfermeiro e de como isso irá refletir no início da carreira profissional destes alunos.

\section{IMPLICAÇÕES PARA A PRÁTICA CLÍNICA}

O desenvolvimento de pesquisas que abordem o tema é fundamental, pois o estresse não é somente um processo resultante da mudança dos hábitos e estilos de vida inadequados, mas, também, um fator de risco para outras doenças e distúrbios psíquicos, podendo ser causador de enfermidades no decorrer da vida.

Além disso, influencia aqueles que convivem com os que sofrem de estresse, como familiares, amigos, colegas de trabalho, que estão envolvidos no desenvolvimento dessa morbidade e, também, no apoio para sua resolução. 
Estudos como este deveriam ser aplicados a outros cursos, para que pudéssemos ter um paradigma da verdadeira situação de como está a saúde mental dos estudantes. A monitorização deste problema deve ser uma prioridade das instituições de ensino superior, de modo a proporcionar uma resposta precoce e adequada a todos os estudantes, que por uma razão ou outra, se encontram com maior vulnerabilidade mental.

Como limitações do estudo, referimos o fato de os resultados não puderem ser generalizado a outros cursos do ensino superior, uma vez que amostra incidiu apenas em estudantes de enfermagem. E também a ausência no questionário proposto de perguntas sobre a situação sócio demográfica, dando a chance de obter dados mais fidedignos acerca da vida desse estudante.

\section{REFERÊNCIAS BIBLIOGRÁFICAS}

Adewuya, A. O. (2006). Prevalence of major depressive disorder in Nigerian college students with alcohol-related problems.General Hospital Psychiatry, 28, 169-173.

Conselho Nacional de Saúde. (2006). Diretrizes e normas regulamentadoras de pesquisas envolvendo seres humanos. Rio Grande do Sul: Universidade Federal do Rio Grande do Sul. Disponível em http://www.bioetica.ufrgs. br/res19696.htm

Comissão Nacional para a Reestruturação dos Serviços de Saúde Mental. (2007). Relatório da Comissão Nacional para a Reestruturação dos Serviços de Saúde Mental. Disponível em http://www.hmlemos.min-saude.pt/docs/ PNacSM2007.pdf

Carvalho, J. C.,e Sequeira, C. (2009). Tradução para a população portuguesa do Questionário de Saúde Mental Positiva (QSM+). In C. Sequeira, J. C. Carvalho, I. Ribeiro, T. Martins, e T. Rodrigues (Eds.), Qualidade de vida em análise (pp. 303-314). Porto: Escola Superior de Enfermagem do Porto.

Castillo, L. G., \& Schwartz, S. J. (2013). Introduction to the special issue on college student mental health. Journal of Clinical Psychology, 69(4), 291-297.

Dela Coleta, J. A., e Dela Coleta, M. F. (2006). Felicidade, bem-estar subjetivo e comportamento acadêmico de estudantes universitários. Psicologia em Estudo, 11(3), 533539. Disponível em http://www.scielo.br
Dias, H. Z. J., Rubin, R., Dias, A. V., e Gauer, G. J. C. (2007). Relações visíveis entre pele e psiquismo: Um entendimento psicanalítico. Revista Psicologia Clínica, 19(2), 23-34.

Eisenberg, D., Gollust, S. E., Golberstein, E., \& Hefner, J. L. (2007). Prevalence and correlates of depression, anxiety, and suicidality among university students. American Journal of Orthopsychiatry, 77(4), 534-542.

Gallagher, R. P. (2013). National survey of college counseling centers, section three: Counseling center clinicians. Pittsburgh: University of Pittsburgh.

Giglio, J. S. (1976).Bem estar emocional em estudantes universitários: um estudo preliminar. $188 \mathrm{f}$. Tese (Doutorado em Psicologia Médica) - Universidade Estadual de Campinas, Campinas, 1976. Disponível em http://libdigi. unicamp.br

Hunt, J.,\&Eisenberg, D. (2010). Mental health problems and help-seeking behavior among college students. The Journal of Adolescent Health: Official Publication of the Society for Adolescent Medicine, 46(1), 3-10.

Jahoda, M. (1958). Currentconceptsof positive mental health. New York: Basic Books.

Lluch, M. T. (2003). Construcción y análisis psicométrico de un cuestionario para evaluar la salud mental positiva. Barcelona: Universidad de Barcelona.

Organização Mundial de Saúde. (2002). Relatório mundial da saúde. Saúde mental: Nova concepção, nova esperança. Disponível em http://www.who.int/whr/2001/en/whr01_ ch1_po.pdf

Ribeiro, J. L. P. (2001). Mental Health Inventory: Um estudo de adaptação à população portuguesa. Psicologia, Saúde \&Doenças, 2(1), 77-99.

Sá, L. (2010). Saúde mental versus doença mental. InC. Sequeira, e L. Sá (Eds,), Do diagnóstico à Intervenção em saúde mental. Porto: Sociedade Portuguesa de Enfermagem de Saúde Mental.

Sequeira, C. A. C., Sá, L., Carvalho, J. C. M., Borges, E. M. N., e Sousa, C. N. (2009).Avaliação da saúde mental positiva em estudantes do ensino superior.Revista Científica da Unidade de Investigação em Ciências da Saúde: Enfermagem. Coimbra: Escola Superior de Enfermagem de Coimbra. 